\title{
Early Receptor Potentials of Rods and Cones in Rodents
}

\author{
WILLIAM L. PAK and THOMAS G. EBREY
}

From the Departments of Biophysics and Physics, The University of Chicago. Dr. Pak's present address is the Department of Biological Sciences, Purdue University, Lafayette, Indiana

\begin{abstract}
The second phase (negative peak) of the early receptor potential of cones has been studied in the all-cone eyes of the Mexican and antelope ground squirrels (Citellus mexicanus and Citellus leucurus) and compared with responses from the rod-dominant eyes of the rat and flying squirrel (Glaucomys volans). The responses obtained from the all-cone eyes tended to be smaller in amplitude, to have higher thresholds, and to be considerably more resistant to light adaptation than the responses from the rod-dominant eyes. The wave forms and time courses of the two types of responses were similar, although the cone potential tended to be less sensitive to temperature variations and its time constants tended to be shorter than those of the rod potential. The spectral sensitivity of the second phase of the early receptor potential of the Mexican ground squirrel closely follows the absorption spectrum of a Dartnall nomogram pigment having its absorption maximum at $540 \mathrm{~m} \mu$. Moreover, as in the case of the rat, the amplitude of the response appears to be linearly related to the amount of pigment bleached in a flash. Thus, in both all-rod and all-cone systems the early receptor potential appears to arise in the photoexcitation of the respective visual pigment and appears to be closely linked to the initial photochemical events. The similarity of the wave form, time course, and stimulus-response curves in the two systems suggests that the early receptor potential is produced by similar mechanisms in all-rod and all-cone systems.
\end{abstract}

\section{IN TRODUGTION}

The possibility that the early receptor potential may be closely linked to the initial events in the photoexcitation of vertebrate visual receptors has prompted intensive studies of the properties of this response (1-5). Most of these studies have been performed either on mixed (rod and cone) eyes (1, 3, 6 ) or on the essentially all-rod eye of the rat (3-5). Although the early receptor potential has been demonstrated in both rods and cones (1-3), no systematic studies of the response in the all-cone eyes have yet been reported. The present work was undertaken in order to study the early receptor potential in all-cone eyes and to compare the response in cones with that in rods. The properties of 
responses in which possible similarities or dissimilarities were looked for included the wave form and its temperature dependence, the spectral sensitivity, and the stimulus-response relationship.

\section{MATERIALS AND METHODS}

Most of the existing information on the early receptor potential of rods comes from the essentially all-rod retina of the albino rat. As it happens, the only mammals known to have pure-cone retinas also belong to a family of rodents, the Sciuridae. The retinas of one member of the Sciuridae, the ground squirrel, are said to be definitely pure cone (7-9). The flying squirrels, on the other hand, are nocturnal and are said to have nearly all-rod retinas $(8,9)$. In this work two species of ground squirrel were examined: the Mexican ground squirrel (Citellus mexicanus) and the white-tailed antelope ground squirrel (Citellus leucurus). For purposes of comparison, essentially all-rod retinas of the albino rat (Sprague-Dawley) and flying squirrel (Glaucomys volans) were also examined, all under nearly the same experimental conditions.

The experimental arrangement and techniques have been described previously $(4,5)$. The responses were obtained from excised eyes by means of the conventional electroretinographic techniques using gross electrodes. Throughout the experiment only fully dark-adapted, freshly excised eyes have been used. The stimulus source consisted of a 60 joule photographic strobe lamp having a flash duration of less than $0.7 \mathrm{msec}$. Artifacts were minimized by housing the lamp in a soft steel box, carefully shielding the electrodes from the flash, and housing the entire apparatus within a Faraday cage. The spectral sensitivities were measured using a set of five BairdAtomic interference filters transmitting at 420,450,500,560, and $620 \mathrm{~m} \mu$. The band width of each filter at $10 \%$ of its peak transmission was found to be about $\pm 6 \mathrm{~m} \mu$.

\section{RESULTS}

\section{Wave Form}

The wave forms of the early receptor potentials of the ground squirrel, flying squirrel, and rat eyes were observed at temperatures between $35^{\circ}$ and $0^{\circ} \mathrm{C}$. The signals obtained at $35^{\circ}, 25^{\circ}$, and $0^{\circ} \mathrm{C}$ in the eyes of the Mexican and antelope ground squirrels are shown in Figs. 1 and 2, respectively. The signals obtained in the eyes of the rat and the flying squirrel under very similar experimental conditions are shown, respectively, in Figs. 3 and 4 . In each figure the trace labeled " $\mathrm{A}$ " corresponds to the response obtained at about $35^{\circ} \mathrm{C}$, and the traces labeled " $\mathrm{B}$ " and " $\mathrm{C}$ " correspond to responses at $25^{\circ}$ and $0^{\circ} \mathrm{C}$, respectively.

Under given experimental conditions much larger signals were obtained from the rod-dominant eyes than from the all-cone eyes, and the threshold of the response of the rod-dominant eyes, as determined by the experimental noise level, was found to be about one log unit lower than that of the responses from the pure-cone retinas. Furthermore, under repeated or prolonged ex- 
posure to the light stimulus the rod responses are reduced or abolished much more readily than the cone responses. These observations are consistent with the expected differences in sensitivity of rods and cones to light stimulus. However, no attempt has been made to quantitatively compare the light sensitivities of rods and cones from these results, since, except in the case of the rat (10), the amount of light absorbed by the visual pigment is uncertain.
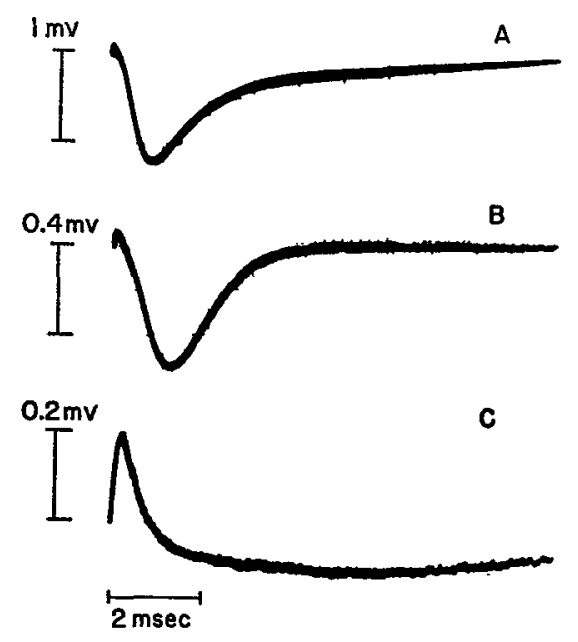

Figure 1. Temperature dependence of the wave form of the early receptor potential elicited from the dark-adapted, excised eye of the Mexican ground squirrel. All cone. A, $35^{\circ} \mathrm{C} ; \mathrm{B}, 25^{\circ} \mathrm{C}$; $\mathrm{C}, 0^{\circ} \mathrm{C}$.

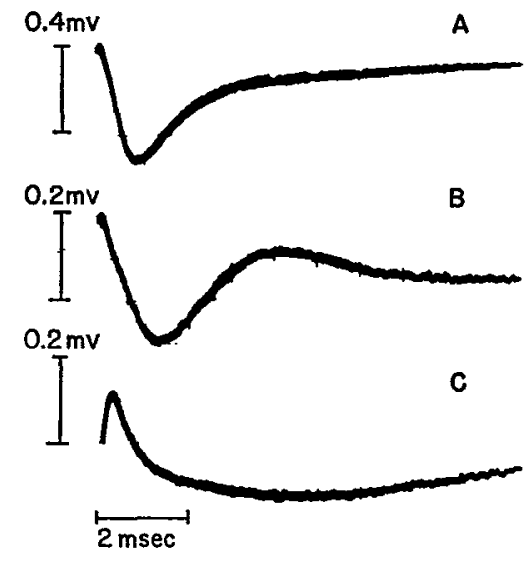

Figure 2. Temperature dependence of the wave form of the early receptor potential elicited from the dark-adapted, excised eye of the antelope ground squirrel. All cone. $\mathrm{A}, 35^{\circ} \mathrm{C} ; \mathrm{B}, 25^{\circ} \mathrm{C} ; \mathrm{C}, 0^{\circ} \mathrm{C}$.

As may be seen in Figs. 1 through 4, the gross features of the wave forms of the early receptor potentials in the four animals examined are similar. In all four cases only a suggestion of the initial phase (positive peak) of the early receptor potential is present near the physiological temperature (Figs. $1 \mathrm{~A}$, $2 \mathrm{~A}, 3 \mathrm{~A}$, and $4 \mathrm{~A}$ ). As the eye is cooled, the amplitude of the second phase (negative peak) decreases and the first phase (positive peak) becomes increasingly more prominent (Figs. 1 to 4 ). 
However, certain differences do appear to exist in the wave forms of the early receptor potentials of rods and cones. In our temperature ranges it was particularly convenient to study the second phase. In the four animals examined, the second phase appears to have a shorter time course in the all-cone retinas than in all-rod retinas. Thus, near the physiological temperature the
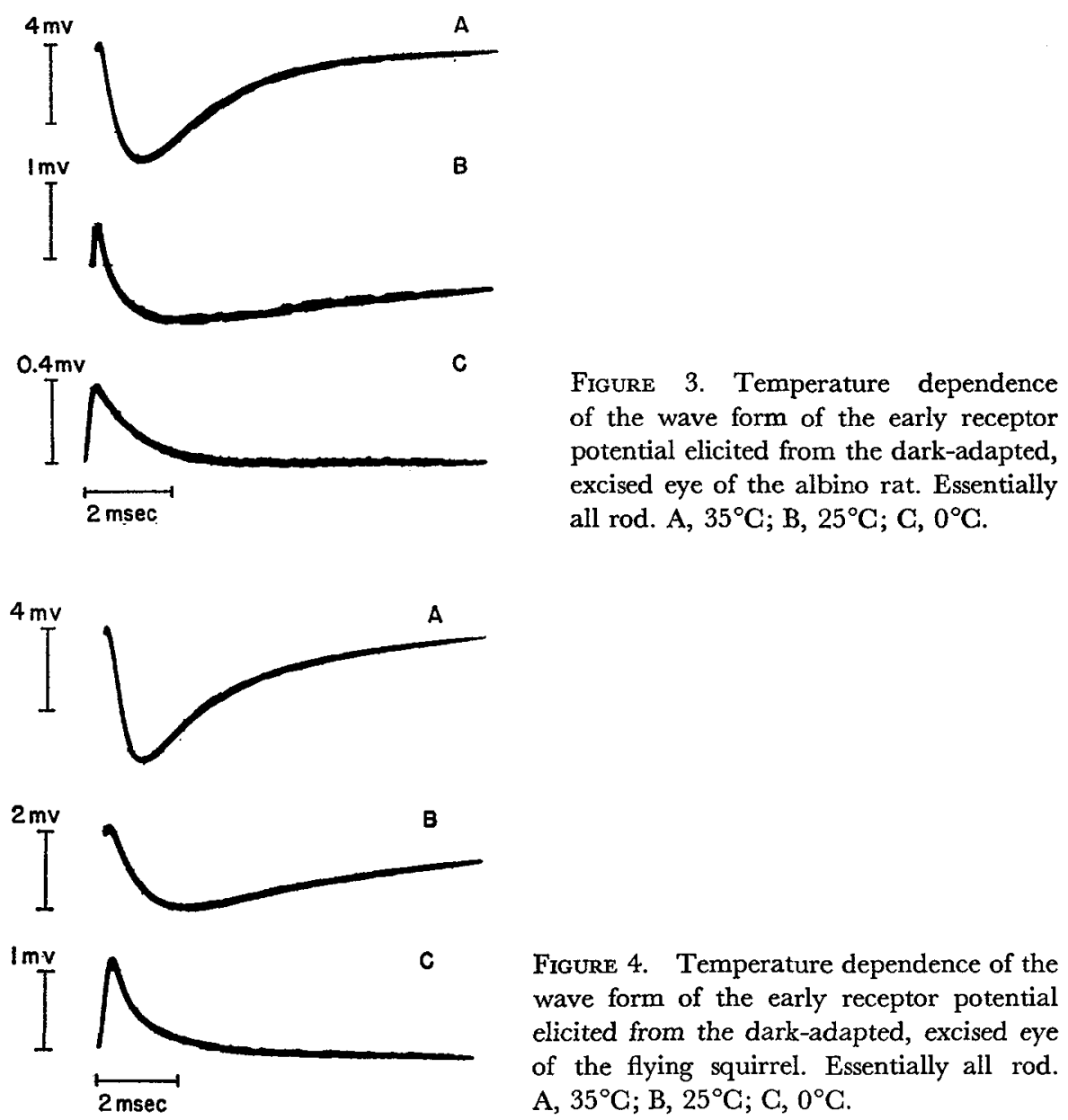

FIGURE 4. Temperature dependence of the wave form of the early receptor potential elicited from the dark-adapted, excised eye of the flying squirrel. Essentially all rod. $\mathrm{A}, 35^{\circ} \mathrm{C} ; \mathrm{B}, 25^{\circ} \mathrm{C} ; \mathrm{C}, 0^{\circ} \mathrm{C}$.

amplitude maximum of the second phase is reached somewhat earlier in the all-cone eyes (Figs. $1 \mathrm{~A}$ and $2 \mathrm{~A}$ ) than in all-rod eyes (Figs. 3A and $4 \mathrm{~A}$ ). Moreover, the cone responses tend to decay more rapidly than the rod responses. In the Mexican and antelope ground squirrels the average half-times of decay of the second phase at $35^{\circ} \mathrm{C}$ are 1.2 and $1.3 \mathrm{msec}$, respectively. The corresponding values for the rat and the flying squirrel are both $1.8 \mathrm{msec}$ under our experimental conditions. It is interesting to note that similar differences in 
time constants have been observed in the later retinal potentials ( $a-$ and $b-$ waves) of rods and cones (11).

As the temperature of the eye is lowered, the time constants of the second phase increase and the amplitude decreases. The effects of cooling, at least among these four animals, are much more pronounced in the rod-dominant eyes than in all-cone eyes. Thus, the differences in time constants between rod responses and cone responses become much more pronounced at lower temperatures. Moreover, in rod-dominant eyes the second phase is suppressed by cooling much more readily than in all-cone eyes and is abolished near $0^{\circ} \mathrm{C}$ (Figs. $3 \mathrm{C}$ and $4 \mathrm{C}$ ), whereas in the ground squirrel eyes the second phase is still present at this temperature (Figs. $1 \mathrm{C}$ and $2 \mathrm{C}$ ).

\section{Stimulus-Response Relationship}

It has been shown that in the all-rod eyes of the albino rat the amplitude of the second phase of the early receptor potential is linearly proportional to the amount of pigment bleached by a flash (3). In the same animal the first phase also has been found to display a similar linear relationship (4). This linear relationship has been interpreted as suggesting a very close coupling between the generation of the response and the photoexcitation of the visual pigment. It is therefore of interest to see whether a similar stimulus-response relationship exists in the all-cone eyes of the ground squirrel.

We present in Fig. 5 the amplitude of the second phase of the early receptor potential obtained in the Mexican ground squirrel at about $35^{\circ} \mathrm{C}$ as a function of the flash energy on a double log plot. The amplitude of the response is given in millivolts, and the flash energy, in units of the threshold energy. A straight line of unit slope fits the experimental data for the first $1.0 \mathrm{log}$ unit or so above the threshold. At higher flash energies the experimental points begin to deviate from the straight line and start to form a plateau. However, this response differs from that in the rat, in that a full plateau is not obtained even with the highest flash energies available. Otherwise the curve we obtain is similar to that obtained by Cone (3) for the all-rod retinas of the albino rat. Thus, it appears that the amplitude of the early receptor potential of cones may also be linearly related to the number of visual pigment molecules excited or bleached in a flash.

\section{Spectral Sensitivity}

One of the more obvious differences to be expected in all-rod and all-cone responses is in their respective spectral sensitivity. The early receptor potential tended to be small in the live squirrel. Therefore, the spectral sensitivity of the second peak of the early receptor potential was measured on excised eyes of the Mexican ground squirrel at a temperature of about $35^{\circ} \mathrm{C}$ by measuring the stimulus intensities needed to elicit equal amplitude responses at five different 
wavelengths. No attempt was made to measure the spectral sensitivity of the first phase.

The data were corrected for the spectral distribution of the stimulus flash and the spectral absorption of the ground squirrel lens. The results are plotted in Fig. 6 along with the spectral sensitivities of the two peaks of the early receptor potential and the $b$-wave of the rat $(3,4,12)$. The errors indicated on

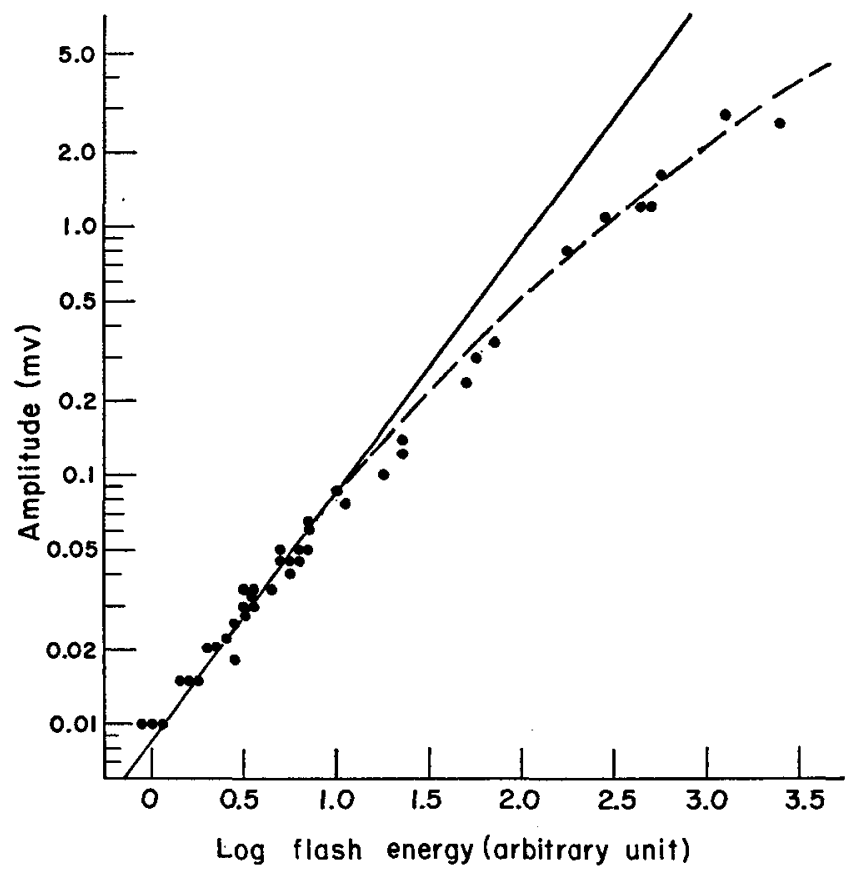

FIGURE 5. Stimulus-response relationship of the second phase of the early receptor potential in the Mexican ground squirrel at about $35^{\circ} \mathrm{C}$. The amplitude of the second phase of the early receptor potential in the all-cone eye of the Mexican ground squirrel is plotted against the stimulus intensity on a double log plot. The amplitude is given in millivolts, and the stimulus intensity in units of the threshold intensity. The straight line represents a line of unit slope.

the cone spectrum points represent statistical errors and do not include any systematic errors that may arise in the correction procedures or in the optical arrangement. The dashed line is the absorption spectrum for a Dartnall nomogram pigment having its absorption maximum at $540 \mathrm{~m} \mu$ (13). The agreement between the experimental points and the calculated curve is satisfactory.

A few years ago Tansley et al. (14) published spectral sensitivity curves based on the b-waves of seven species of diurnal squirrels. Their curves showed two maxima at about 535 and $490 \mathrm{~m} \mu$. They found that two typical curves could be obtained from the same animal under the same experimental conditions, 
each having one of the maxima markedly higher than the other. The appearance of one type of curve rather than the other appeared to be entirely unrelated to any experimental conditions that they could control. Such "shifting of peaks" as that observed by these workers in the action spectrum of the b-

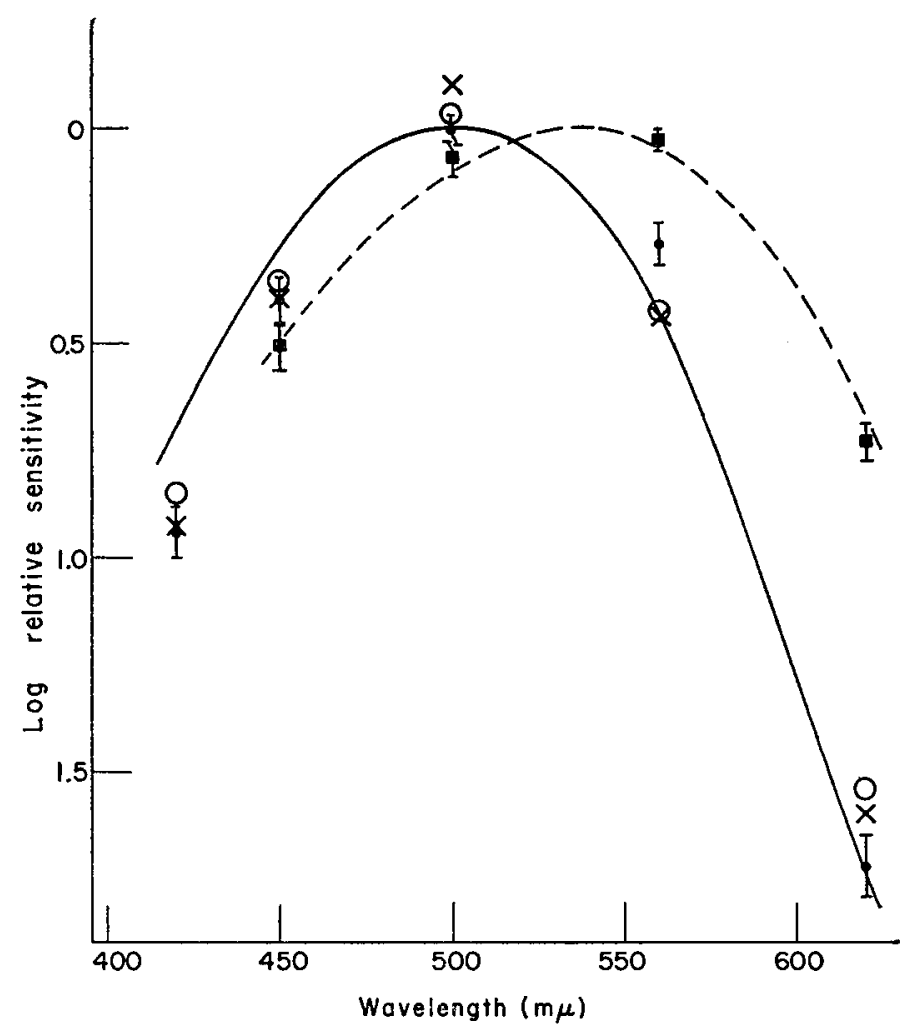

FIGURE 6. Spectral sensitivities of the early receptor potentials in rod-dominant eyes and in pure-cone eyes. Solid circles, spectral sensitivity of the first phase in the albino rat (reference 4); vertical bars indicate statistical errors. Crosses, spectral sensitivity of the second phase in the albino rat (reference 3). Open circles, spectral sensitivity of the b-wave in the albino rat (reference 3 ). Solid line, spectral sensitivity of the b-wave of the albino rat obtained by Dodt and Echte (reference 12). Squares, spectral sensitivity of the second phase in the all-cone retina of the Mexican ground squirrel; vertical bars indicate statistical errors. Dashed line, absorption spectrum of a Dartnall nomogram pigment having its absorption maximum at $540 \mathrm{~m} \mu$.

wave was not seen in the action spectrum of the early receptor potential. Our results are consistent with that set of curves in which the $535 \mathrm{~m} \mu$ peak is high. On the other hand, because of the high stimulus intensity required to elicit the early receptor potential, it was not possible to explore the presence of the secondary peaks in the action spectrum of the early receptor potential with our present setup. 
Also, the absorption spectrum of the visual pigment in the retina of the thirteen-lined ground squirrel (Citellus tridecemlineatus) has been measured by Dowling and Brown by means of microspectrophotometry (7). The maximum of the spectrum was found to be $523 \mathrm{~m} \mu$ and the curve was slightly narrower than that of a Dartnall pigment.

\section{DISCUSSION}

The results of the present studies indicate that the second phase (negative peak) of the early receptor potentials of rods and cones display essentially similar properties. In both cases a linear stimulus-response relationship has been obtained. The temperature dependence of the wave form is also similar. In both cases lowering the temperature of the eye tends to preferentially reduce the amplitude of the second phase, making the first phase more prominent. However, cooling appears to affect the second phase of rods much more readily than the corresponding cone response.

Recently, Brown (15) reported that an early potential having a polarity and time course similar to those of the second phase of the early receptor potential can be obtained from the pigment epithelium-choroid complex of the toad. Unlike the early receptor potential, the new potential was found to be highly resistant to light adaptation.

Conceivably a part of the differences in wave forms of the early receptor potentials of the four animals examined may be due to different degrees of contamination by the new potential. In the case of the rat, however, any contamination of the early receptor potential by a photostable component, such as the new potential obtained in the pigment epithelium-choroid complex, if present at all appears to be negligibly small at our stimulus intensity, since the response obtained in the whole eye can be readily abolished or reduced to a negligible size by repeated stimuli (16). In the case of the Mexican ground squirrel, on the other hand, a potential similar to that described by Brown can be obtained from the pigment epithelium-choroid complex. However, neither the amplitude nor the peak time of this potential changes very much with temperature variation between $25^{\circ}$ and $35^{\circ} \mathrm{C}$, whereas the total negative potential undergoes a marked increase in amplitude and decrease in peak time (Fig. 1). It is, therefore, inferred that the dominant negative potential near the physiological temperature in the intact eye of the Mexican ground squirrel is the second phase of the early receptor potential. This conclusion is strengthened by the observation that at $35^{\circ} \mathrm{C}$ about $60 \%$ of the negative potential can be readily abolished in about three or four flashes. A part of the remaining response probably is due to the presence of the pigment molecules which have been rephotoisomerized to the 11-cis form from the intermediate states during the preceding flash sequence. Thus, near the physiological temperature at which most of the present series of experiments were carried out, the contamination by the 
new potential arising from the pigment epithelium-choroid complex appears to be relatively small.

A possible difference in threshold of the early receptor potential and the new potential has been studied in the frog eye by examining the potentials from the excised retina and the eye cup. The threshold of the potential from the pigment epithelium is found to be more than one log unit higher than that of the early receptor potential at room temperatures. Since the action spectrum of the second phase of the early receptor potential was based on near threshold responses, the spectral sensitivity curve of the second phase should be little affected by the presence of the pigment epithelial potential.

It is possible, however, that the observed difference in temperature dependence of the wave forms in the four animals examined may be due to different degrees of contamination by the potential from the pigment epithelium-choroid complex.

The action spectrum of the early receptor potential in the all-cone eye of the ground squirrel clearly indicates that the pigment responsible for the second phase is not rhodopsin. The fact that the spectrum agrees well with Dartnall's nomogram curve peaking at about $540 \mathrm{~m} \mu$ is suggestive of the absorption spectrum of one of the cone pigments (17). Unfortunately, the interpretation of the existing information on the spectral sensitivities of the squirrel retina $(14,18)$ is not clear cut. Nevertheless, our results are consistent with one set of curves obtained by Tansley $e t$ al. (14). It thus appears very likely that the pigment responsible for the second peak in the retina of the Mexican ground squirrel is one or more of the ground squirrel cone pigments.

These results are consistent with the view that both in rods and in cones the second peak of the early receptor potential arises as a consequence of photoexcitation of the respective visual pigment and that the mechanisms of production of the response in the two cases are essentially similar. Furthermore, the fact that the early receptor potential can be elicited from both rods and cones of various vertebrates suggests that the mechanism of its generation is not critically dependent on any structural differences between rods and cones.

We thank Professor John R. Platt for his advice and support.

This work was supported in part by the United States Public Health Service and by a National Science Foundation Science Faculty Fellowship awarded to Dr. Pak.

Received for publication 6 December 1965.

\section{REFERENCES}

1. Brown, K. T., and Murakami, M., A new receptor potential of the monkey retina with no detectable latency, Nature, 1964, 201, 626.

2. Brown, K. T., and Murakami, M., Biphasic form of the early receptor potential of the monkey retina, Nature, 1964, 204, 739. 
3. Cone, R. A., Early receptor potential of the vertebrate retina, Nature, 1964, 204, 736.

4. PAK, W. L., and Cone, R. A., Isolation and identification of the initial peak of the early receptor potential, Nature, 1964, 204, 836.

5. PAK, W. L., and EBrey, T. G., Visual receptor potential observed at sub-zero temperatures, Nature, 1965, 205, 484.

6. PAK, W. L., Some properties of the early electrical response in the vertebrate retina, Cold Spring Harbor Symp. Quant. Biol., 1965, 30, 493.

7. Dowling, J. E., Structure and function in the all-cone retina of the ground squirrel, in Symposium on the Physiological Basis for Form Discrimination, Brown University, Providence, Rhode Island, 1964, 17.

8. TANSLEY, K., Comparative anatomy of the mammalian retina with respect to the electroretinographic response to light, in The Structure of the Eye, (G. K. Smelser, editor), New York, Academic Press Inc., 1961, 193.

9. Walls, G. L., The vertebrate eye, Bull. Cranbrook Inst. Sc., 1942, 19.

10. Cone, R. A., Quantum relations of the rat electroretinogram, J. Gen. Physiol., 1963, 46, 1267.

11. WiRth, A., La durata dello stimolo come mezzo di separazione delle componenti fotopica e scotopica dell'elettroretinogramma, Arch. sc. biol. Bologna, 1956, 11, 163. Granit, R., Neurophysiology of the retina, in The Eye, (H. Davson, editor), New York, Academic Press Inc., 1962, 2, 580.

12. Dodt, E., and Earte, K., Dark and light adaptation in pigmented and white rat as measured by electroretinogram threshold, $J$. Neurophysiol., 1961, 24, 427.

13. Dartnall, H. J. A., The interpretation of spectral sensitivity curves, Brit. Med. Bull., 1953, 9, 24.

14. Tansley, K., Copenhaver, R. M., and Gunkel, R. D., Spectral sensitivity curves of diurnal squirrels, Vision Research, 1961, 1, 154.

15. Brown, K. T., An early potential evoked by light from the pigment epitheliumchoroid complex of the eye of the toad, Nature, 1965, 207, 1249.

16. Ebrey, T. G., and Cone, R. A., Early non-photolabile electrical response of the vertebrate eye, submitted to Nature.

17. Marks, W. B., Visual pigments of single goldfish cones, J. Physiol., 1965, 178, 14. Marks, W. B., Dobelle, W. H., and MacNichol, E. F., JR., Visual pigments of single primate cones, Science, 1964, 143, 1181. Brown, P. K., and WALD, G., Visual pigments in single rods and cones of the human retina, Science, 1964, 144, 45.

18. Arden, G. B., and Tansley, K., The spectral sensitivity of the pure-cone retina of the souslik (Citellus citellus), J. Physiol., 1955, 130, 225; The spectral sensitivity of the pure-cone retina of the grey squirrel (Sciurus carolinensis leucotis), J. Physiol., 1955, 127, 592. 